\title{
Évolution de l'organisation du système vocalique du français en contexte de bilinguisme simultané
}

\author{
Marie Philippart de Foy ${ }^{1}$, Véronique Delvaux ${ }^{1,2}$, Kathy Huet ${ }^{1}$, Morgane Monnier ${ }^{1}$, Myriam \\ Piccalugal et Bernard Harmegnies ${ }^{l}$ \\ ${ }^{1}$ Institut de Recherche en Sciences et Technologies du Langage, Université de Mons, Belgique \\ ${ }^{2}$ Fonds National de la Recherche Scientifique, Bruxelles, Belgique.
}

Résumé. Cette étude vise à examiner le développement phonéticophonologique du français et plus précisément la progressive organisation du système vocalique chez des enfants bilingues préscolaires exposés à l'une des trois combinaisons linguistiques suivantes: français-italien, français-arabe et français-mandarin. Les productions orales des enfants ont été recueillies longitudinalement via une tâche de dénomination originale en français. La dominance linguistique des enfants ainsi que leur développement lexical ont été évalués via des questionnaires parentaux. L'évolution de l'organisation du système vocalique a été évaluée via l'indice PHI (Huet \& Harmegnies, 2000) permettant de générer une mesure de dispersion des valeurs formantiques dans l'espace vocalique. L'impact de la combinaison et de la dominance linguistiques ainsi que du développement lexical sur l'organisation du système vocalique a été investigué. Les résultats indiquent: (1) un système vocalique initialement mieux organisé chez les bilingues français-italien, (2) une augmentation parallèle de l'organisation du système vocalique et de la compétence lexicale chez les bilingues français-italien et français-arabe et (3), aucun effet de la dominance linguistique. Ces résultats enrichissent les connaissances sur le développement de la parole en français dans des contextes de bilinguisme simultané contrastés.

\begin{abstract}
Evolution of the organization of the French vocalic system in the context of simultaneous bilingualism. The current study aims at examining the phonetic and phonological development of French and more particularly, the progressive organization of the vocalic system in bilingual toddlers exposed to one the three following language pairs: French-Italian, French-Arabic and French-Mandarin. The children's speech productions in French have been longitudinally collected via an original picture-naming task. The linguistic dominance and the lexical development have been assessed with parental questionnaires. The evolution of the vocalic system's organization has been examined through the use of the PHI index (Huet \& Harmegnies, 2000) allowing to generate a dispersion measure of the formant values in the vocalic space. The impact of the language pair, the linguistic dominance and the lexical development on the organization of the vocalic system has been investigated. The results show: (1) a vocalic system initially better organized in French-Italian bilinguals, (2) a parallel increase of the organization of the vocalic system and of the lexical competence in both French-Italian and French-Arabic bilinguals and (3), no effect of the linguistic dominance. These findings provide new insights
\end{abstract}


about French speech development in different contexts of simultaneous bilingualism.

\section{Introduction}

Les connaissances dont on dispose actuellement sur l'acquisition des compétences phonétiques et phonologiques en français par les enfants en situation de bilinguisme précoce demeurent aujourd'hui encore assez limitées, particulièrement en ce qui concerne l'installation de la maîtrise de la production des voyelles. De fait, le français a fait l'objet d'un nombre restreint d'études portant sur l'acquisition de sa phonologie en contexte bilingue (Paradis, 2001 ; Brulard \& Carr, 2003 ; MacLeod et al., 2011 ; Almeida, 2011 ; Kehoe \& Havy, 2019) et les travaux existants se sont majoritairement focalisés sur la production des consonnes (Brulard \& Carr, 2003 ; MacLeod et al., 2011 ; Almeida, 2011). Par ailleurs, les recherches ont principalement étudié le bilinguisme français-anglais (Paradis, 2001 ; Brulard \& Carr, 2003 ; MacLeod et al., 2011). A notre connaissance, seule une étude menée par Kehoe et Havy (2019) a investigué la production de voyelles chez des enfants bilingues francophones préscolaires tout en incluant plusieurs combinaisons linguistiques associant le français à différentes langues. Les voyelles y ont fait l'objet d'observations perceptuelles débouchant sur des mesures de précision de réalisation basées sur des transcriptions phonétiques réalisées par des experts humains, sans recours à l'analyse objective des aspects acoustiques du signal. L'absence d'analyses basées sur des données acoustiques peut s'avérer particulièrement problématique dans le cadre de l'étude de la parole enfantine qui est caractérisée par une grande variabilité, voire une certaine atypicité, et qui, de ce fait, est propice à déclencher chez l'auditeur des réflexes de reconstruction et d'interprétation plutôt que d'analyse (Haidar, 2018).

Si l'acquisition des voyelles a été également peu étudiée chez les enfants monolingues francophones, quelques travaux incluant des analyses acoustiques peuvent toutefois être mentionnés. D'une part, des études centrées sur la comparaison entre enfants normoentendants et sourds d'âge scolaire ont impliqué des analyses des valeurs formantiques et/ou de l'espace vocalique sur base des voyelles périphériques /a, i, u/ (Ryalls, Larouche \& Giroux, 2003) ou de toutes les voyelles orales du français (Grandon, 2016) suggérant une stabilisation de la catégorisation des voyelles entre 5 et 11 ans. D'autres études focalisées sur le développement et les différences inter-linguistiques des espaces vocaliques de nourrissons canadiens francophones et anglophones âgés entre 10 et 18 mois (Rvachew et al., 2006 ; 2008) ont montré une influence précoce de la langue sur les valeurs formantiques ainsi qu'une expansion de l'espace vocalique liée à une occurrence croissante des voyelles fermées $/ \mathrm{i} /$ et $/ \mathrm{u} /$ dans le babillage avec l'âge. Il existe donc actuellement encore très peu de données objectivées sur les caractéristiques acoustiques des voyelles du français chez les enfants d'âge préscolaire, particulièrement en contexte d'acquisition bilingue. En outre, il s'avère également qu'on est fort dépourvu de connaissances concernant la dynamique d'évolution des compétences phonétiques et phonologiques, tant chez l'enfant monolingue que chez l'enfant en situation de bilinguisme précoce. Certes, des observations enracinées dans la clinique orthophonique ont apporté des informations sur certains mécanismes développementaux, en particulier les « processus phonologiques simplificateurs » (Maillart, 2006). Ces investigations se focalisent généralement sur un phonème ou un groupe de phonèmes, laissant souvent de côté l'aspect systémique du développement. D'autre part, le contexte clinique de la plupart de ces réflexions induit naturellement un regard extemporané, centré sur l'ici et maintenant du phénomène, qui laisse peu de place à une réflexion sur la dynamique de l'évolution. 
Par ailleurs, la plupart des recherches tentant une approche génétique optent, par souci de facilité, pour des paradigmes transversaux (où des sujets différents relevant de tranches d'âges successives sont étudiés) plutôt que pour des paradigmes longitudinaux (où chaque sujet est observé de manière répétitive), ce qui, commente Vion (1987), conduit à une perspective plus descriptive que dynamique et à privilégier la mise au jour de comportements dominants, au détriment des stratégies individuelles d'appropriation du système phonologique. Par ailleurs, comme le note Kail (1983), la question de la possible influence des structures de la langue à acquérir sur le processus d'apprentissage lui-même a été débattue à divers égards (aux plans phonologique, lexical et syntaxique à tout le moins). Si on en admet le principe, il peut apparaître censé de penser que, dans la mesure où l'enfant bilingue est simultanément exposé à deux systèmes, chacun de ceux-ci est susceptible d'exercer ses effets propres séparément ou en interaction (Flege, 2007 ; Paradis \& Genesee, 1996 ; Fabiano-Smith \& Barlow, 2010). Dès lors, les ressemblances et/ou différences structurelles entre ces systèmes peuvent être pointées comme facteur de variation possible entre les profils de développement. D'autre part, en fonction de la prégnance relative de l'une ou l'autre des deux langues dans l'environnement linguistique de l'enfant, il se pourrait que les effets de la structure d'une des langues soient plus importants que ceux de la structure de l'autre langue ; la dominance linguistique apparaît donc comme une variable à prendre en considération.

S'inscrivant dans une démarche empirique, l'étude exploratoire que nous présentons ici a pour objectif de contribuer à combler les lacunes que présente la littérature concernant l'évolution de la production des voyelles du français chez l'enfant en situation de bilinguisme précoce. Un premier aspect innovant de l'étude consiste à adopter une approche comparative en observant des enfants exposés à différentes combinaisons linguistiques incluant toutes le français et une deuxième langue dont le degré de similarité avec le français varie. Il s'agit plus précisément des trois combinaisons suivantes : (1) français-italien, (2) français-arabe et (3) français-mandarin. L'objectif est d'observer si le degré de proximité entre les langues en contact influence le développement phonéticophonologique en français, et plus particulièrement la structuration du système vocalique, et si le fait d'acquérir deux systèmes plus ou moins similaires aurait un effet plus ou moins facilitateur pour l'acquisition vocalique en français. Un objectif parallèle est d'examiner l'impact du degré d'exposition aux deux langues (i.e., la dominance linguistique) ainsi que du développement lexical des enfants sur la progressive organisation du système vocalique. Afin de mener l'étude à bien, nous développons un protocole original de recueil des productions orales des enfants et une nouvelle méthodologie d'analyse quantitative des données acoustiques, fondée sur l'utilisation originale dans ce contexte de l'indice PHI (Huet \& Harmegnies, 2000), dont la fonction est de quantifier le degré d'organisation des systèmes vocaliques.

\section{Méthode}

\subsection{Participants}

Les sujets constituent un échantillon occasionnel composé de 18 enfants bilingues d'âge préscolaire ( 8 filles et 10 garçons), résidant en Belgique (principalement à Bruxelles), observés à quatre reprises au cours d'une année et initialement âgés entre 21 et 36 mois (âge moyen=34 mois, E.T.=7 mois). Ils se caractérisent par leur appartenance à l'une des trois combinaisons linguistiques suivantes : français-italien, français-arabe et françaismandarin. La répartition des participants dans chaque groupe linguistique est la suivante : 11 bilingues français-italien ( 5 filles et 6 garçons, âge moyen global $=34$ mois, E.T. $=7$ 
mois), 5 bilingues français-arabe ( 2 filles et 3 garçons, âge moyen global $=34$ mois, E.T. $=8$ mois) et 2 bilingues français-mandarin ( 1 fille et 1 garçon, âge moyen global = 37 mois, E.T. $=5$ mois). Exposés à leurs deux langues depuis la naissance (ou dès les premiers mois), les participants sont des bilingues simultanés présentant des degrés d'exposition similaires aux deux langues, au minimum trois jours entiers par semaine ${ }^{1}$.

\subsection{Recueil des données}

Afin de répondre aux objectifs de recherche, nous avons mis au point un paradigme expérimental spécifique assurant au moins 5 rencontres avec l'enfant et ses proches. Lors du premier contact, nous avons collecté des données hétéro-rapportées via deux questionnaires parentaux. Premièrement, les parents ont rempli un questionnaire élaboré à partir du PABiQ (Tuller, 2011) afin de documenter les spécificités de l'expérience bilingue de chaque participant. Ensuite, ils ont rempli des adaptations des MacArthur-Bates Communicative Development Inventories (Fenson et al., 1993) dans les deux langues de l'enfant (Kern \& Gayraud, 2010 ; Caselli \& Casadio, 1995 ; Tardif et al., 2008) afin d'évaluer le développement lexical en français et globalement, dans les deux langues. Les informations ainsi recueillies ont été actualisées lors de chacune des rencontres ultérieures. Les productions orales en français ont été recueillies à partir de la deuxième rencontre (Session 1, ci-après $\mathrm{S} 1$ ) et lors de trois rencontres suivantes (Session 2, 3 et 4, ci-après S2S3-S4) planifiées à intervalles réguliers de quatre mois. Les enfants ont été enregistrés à leur domicile au moyen d'un enregistreur audio-portable Zoom H5 et d'un micro chant Sennheiser E912 BK. Les productions de parole ont été recueillies via une tâche de dénomination de mots insérée au sein d'un jeu avec un livre imagier afin de cibler des structures phonologiques spécifiques dans un contexte interactif. Les items à faire produire ont été sélectionnés sur base de critères psycholinguistiques et phonologiques listés par ordre d'importance : (1) l'âge d'acquisition (ci-après AoA) sur base des normes de Chalard et al. (2003) et des rapports parentaux de Kern et Gayraud (2010), (2) l'imageabilité des mots $^{2}$, la présence dans le corpus total de (3) tous les phonèmes du français, (4) toutes les consonnes du français en position initiale/médiane/finale dans le mot, (5) de groupes consonantiques (ci-après GC) dans différentes positions dans le mot et (6), de différentes structures syllabiques et longueurs de mots. Le corpus final inclut 3 items d'entrainement, 48 items test et 2 items leitmotiv ([maja] et [wiwi]) représentant des personnages de dessins animés bien connus de tous les enfants (Maya et Oui-Oui) et impliquant des semiconsonnes en position intervocalique. Les items ont été organisés dans un ordre spécifique, en 8 séries de 6 items, par AoA et complexité phonologique croissants, et les items leitmotiv ont été insérés entre chaque série. La complexité phonologique a été évaluée à partir de critères précis afin de générer un classement de complexité des mots. Exposés dans le Tableau 1, ces critères de complexité se situent à différents niveaux phonologiques et possèdent différents degrés auxquels une valeur spécifique a été attribuée.

Tableau 1. Critères de complexité phonologique avec leur valeur assignée entre parenthèses.

\begin{tabular}{|c|c|}
\hline $\begin{array}{l}\text { Niveau de } \\
\text { complexité }\end{array}$ & Critères avec la valeur assignée \\
\hline $\begin{array}{l}\text { Syllabe } \\
\text { CSyl }\end{array}$ & $\begin{array}{l}\text { Structure et longueur des mots : mono-/bisyllabiques avec réduplication (0) vs. } \\
\text { bisyllabiques avec structure variée (1) vs. trisyllabiques (2) }\end{array}$ \\
\hline \multirow{3}{*}{$\begin{array}{l}\text { Segmental } \\
\text { CSeg }\end{array}$} & Absence $(0) v s$. présence de voyelles nasales $(0,5)$ \\
\hline & Absence (0) $v s$. présence des fricatives $/ \mathrm{J} /, / \mathrm{z} /, / 3 /$ en attaque (1) $v s$. coda (2) \\
\hline & Présence d'une initiale vocalique (1) \\
\hline \multirow{2}{*}{$\begin{array}{l}\text { Intersegmental } \\
\qquad C I S\end{array}$} & Absence (0) vs. présence de GC de 2 consonnes (1) en coda (2) \\
\hline & Absence (0) vs. présence de GC de 3 consonnes (2) en coda (3) \\
\hline
\end{tabular}


Pour chaque mot, nous avons calculé un indice de complexité phonologique sur base de l'équation ci-dessous où, pour le mot $i, I C_{i}$ correspond à l'indice de complexité globale, $\mathrm{CSyl}_{i}$ à l'indice de complexité syllabique, $C S e g_{i}$ à l'indice de complexité segmentale, $\operatorname{CIS}_{i}$ à l'indice de complexité intersegmentale et $\max _{j}$ à la plus haute valeur de l'indice parmi tous les mots $j$ du corpus.

$$
I C_{i}=\left(\frac{C S y l_{i}}{\max _{j} C S y l_{i}}+\frac{C S e g_{i}}{\max _{j} \operatorname{CSeg}_{i}}+\frac{C I S_{i}}{\max _{j} C I S_{i}}\right) / 3
$$

La Figure 1 représente les différentes séries de mots dans un graphique cartésien ou l'axe des $\mathrm{X}$ correspond à l'ordre de présentation des mots et l'axe des $\mathrm{Y}$ au degré de complexité phonologique. Chacune des 8 séries apparait dans une couleur spécifique et 3 items d'AoA et de niveau de complexité différents sont mis en exergue (la liste des items classés par ordre de présentation avec leur valeur d'indice de complexité globale figure dans le Tableau 5 en annexe).

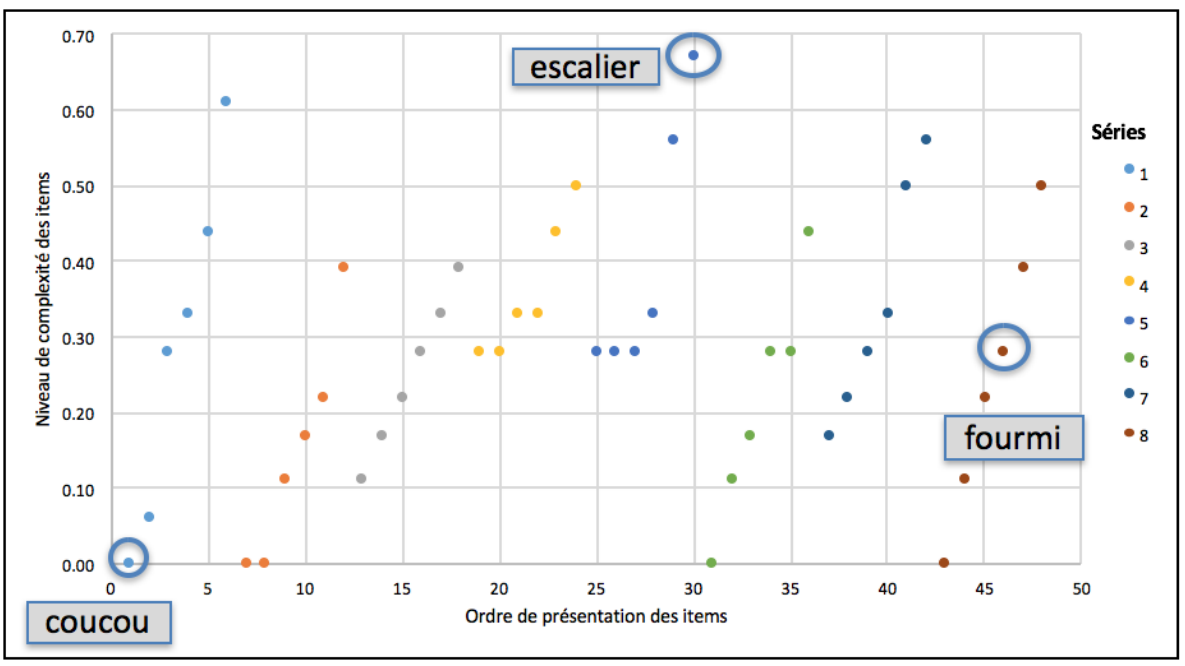

Fig. 1. Graphique représentant l'ordre de présentation des items en fonction de l'AoA et de la complexité.

Cet ordre d'organisation permettait de débuter la tâche avec des mots vraisemblablement connus par les plus jeunes enfants ainsi que de contrebalancer le degré de complexité tout au long du corpus en présentant des productions graduellement plus difficiles, avec de fréquents retours à des productions plus faciles. En outre, lorsque l'enfant se montrait incapable de procéder à une dénomination, d'autres techniques d'élicitation - indiçage sémantique et/ou phonologique $\left(1^{\mathrm{er} / \mathrm{ere}}\right.$ phonème/syllabe $\mathrm{du}$ mot $)$ et répétition ${ }^{3}-$ étaient utilisées. Cette technique de testing adaptatif a permis de maximiser le nombre de productions réalisées par les enfants mais a une contrepartie négative: le fait que l'ensemble des mots produits varie en fonction de l'enfant et de la session.

\subsection{Traitement et sélection des données}

Sur base des réponses au questionnaire parental a été généré un indice de dominance linguistique ou IDL (basé sur l'IDL développé par Almeida et al., 2016) actualisé lors de chaque session. Les valeurs d'IDL ont permis de classer les participants en trois sous- 
groupes selon qu'ils ont: (1) une dominance linguistique en français, (2) une dominance linguistique dans l'autre langue (italien/arabe/mandarin) et (3), un bilinguisme équilibré. Les données obtenues via les adaptations des MBCDI ont permis de calculer, pour chaque participant, deux scores de vocabulaire : un score pour le français et un score global pour les deux langues combinées.

Les enregistrements ont été annotés via PRAAT (Boersma \& Weenink, 2015) dans le format Textgrid sur six couches d'annotation : (1) le locuteur, (2) l'item-cible, (3) la transcription phonétique, (4) la technique d'élicitation (dénomination ou répétition), (5) la séquence de segments alignés formant les mots et (6), les éventuels commentaires sur les productions. Toutes les annotations ont été faites manuellement excepté pour la couche 5 pour laquelle la segmentation et l'alignement automatique ont été réalisés via le logiciel SPPAS (Bigi, 2015) à partir des transcriptions dans la troisième couche d'annotation.

\subsection{Analyses}

Notre protocole nous a permis de récolter longitudinalement des productions des 11

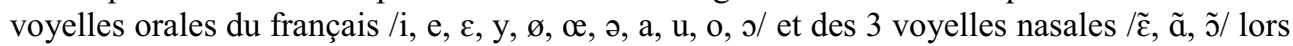
des quatre sessions d'enregistrement de chaque participant. Du fait de la présence en grand nombre des deux items leitmotiv Maya et Oui-Oui (impliquant les voyelles /a, i/ et les semi-consonnes $/ \mathrm{j}, \mathrm{w} /$ ), les trois timbres $[\mathrm{a}, \mathrm{i}, \mathrm{u}]$ sont plus fréquents dans notre corpus. La $5^{\text {ème }}$ couche d'annotation PRAAT impliquant les phonèmes segmentés a servi de base pour les analyses. Les valeurs des trois premiers formants des voyelles mesurées à $50 \%$ de la durée du segment ont été automatiquement extraites via un script PRAAT (Méthode Burg, 3 formants, $5500 \mathrm{~Hz}$ ) et ensuite vérifiées manuellement. Sur base des valeurs formantiques, le degré d'organisation du système vocalique a été évalué via l'indice PHI, une mesure de quantification développée par Huet \& Harmegnies (2000) et utilisée ici pour la première fois pour le traitement de productions enfantines.

Il se définit comme suit :

$$
\phi=\frac{\mathrm{CM}_{\text {inter }}}{\mathrm{CM}_{\text {intra }}}
$$

avec

$\mathrm{CM}_{\text {inter }}$, le Carré moyen des distances euclidiennes entre les centroïdes des nuages vocaliques dans l'espace des points-voyelles

$\mathrm{CM}_{\text {intra }}$, le Carré moyen des distances euclidiennes à l'intérieur des nuages vocaliques dans l'espace des points-voyelles

L'indice PHI a été développé dans le cadre des travaux de Harmegnies et collaborateurs centrés sur la déstructuration des systèmes vocaliques sous l'effet du changement de style de parole chez l'adulte, dans diverses langues romanes (Harmegnies \& Poch-Olive, 1992 ; Blecua-Falgueras, Poch-Olive \& Harmegnies, 1993; Delplancq, Harmegnies \& Poch-Olive, 1994; Harmegnies \& Poch-Olive, 1994). Inspiré de l'analyse de variance, il exprime en un rapport la relation existant, dans le plan formantique, entre les distances euclidiennes interet intra-clusters vocaliques (voir Figure 2). 


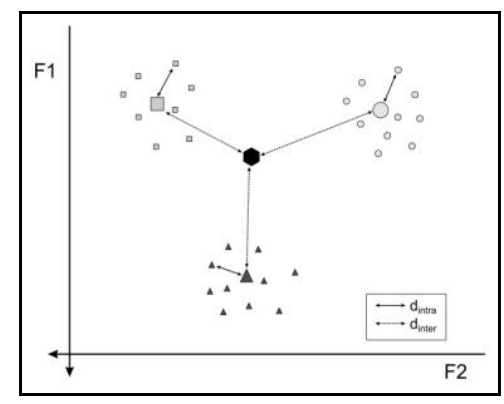

Fig. 2. Principe de construction de l'indice PHI sur base de trois nuages vocaliques dans un plan F1-F2.

Si les distances entre nuages de points sont grandes en comparaison des distances au sein des nuages de points, PHI a une valeur importante. Si les nuages de points sont proches les uns des autres et/ou chacun fort étalés dans le plan et/ou si les nuages sont mal séparés, le rapport entre les distances inter et intra diminue et PHI décroît. Un PHI élevé est donc indiciaire d'une bonne séparation entre les nuages de points et, ipso facto, d'une structuration phonique ne laissant pas de place aux confusions inter-phonémiques. Plus PHI décroît, plus devient probable la possibilité de confusions inter-phonémiques. L'indice fait donc figure de mesure d'entropie du système vocalique (Harmegnies \& Poch-Olive, 1992), ou autrement dit, est révélateur de son degré d'organisation.

Si l'on voit le développement langagier de l'enfant comme un processus de structuration graduelle de ses espaces linguistiques, l'indice PHI peut dès lors apparaître comme un candidat intéressant à l'appréciation du degré de structuration du système phonique. Une réflexion d'une autre nature peut être couplée à ce raisonnement. Contrairement aux populations adultes, les enfants présentent des caractéristiques anatomiques évolutives. Leurs structures articulatoires connaissent de la naissance à la fin de l'âge préscolaire d'importantes modifications sur le plan anatomique, tels que la descente du larynx et l'accroissement de la taille des articulateurs supra-glottiques. Des changements considérables peuvent donc être attendus, notamment en termes de résonance: les résonateurs gagnant en volume, les longueurs d'onde privilégiées s'accroissent et les fréquences diminuent. Conséquemment, les plages de variation dans l'espace formantique se réduisent (Boë et al., 2008). L'enfant est donc le lieu de deux tendances antagonistes : celle, d'une part, qui limite naturellement, du fait de sa croissance, l'espace acoustique de ses productions et celle, d'autre part, qui l'amène à faire un effort de précision articulatoire lui permettant de construire un système acoustique de mieux en mieux différencié dans un espace de variations fréquentielles de plus en plus réduit. Les valeurs fréquentielles des formants observées dans le cadre de l'analyse des productions résultent donc du jeu complexe de ces étiologies. Pour en extraire l'effet du seul contrôle par l'enfant, il faudrait pouvoir disposer du suivi précis de ses modifications anatomiques afin d'en neutraliser l'effet. Ceci est cependant très difficile à mettre en œuvre : il faudrait en effet pouvoir soumettre les sujets à des examens d'imagerie médicale réguliers. Plutôt qu'une centration sur les tendances centrales des valeurs formantiques, nous avons donc préféré une focalisation sur leurs variabilités, et même, plus précisément, sur les rapports de ces variabilités. Ceci constitue une autre raison du choix de l'indice PHI, vu ses bases conceptuelles. Nous allons donc l'utiliser en tant qu'outil permettant d'évaluer le degré de structuration du système vocalique de l'enfant.

Etant donné que les enfants n'ont pas nécessairement produit toutes les voyelles du français lors de chaque session (soit parce qu'ils n'ont pas produit certains items ou parce qu'ils ont 
élidé une syllabe) nous restreindrons l'application de l'indice PHI aux seules voyelles cardinales $(/ \mathrm{a}, \mathrm{i}, \mathrm{u} /)$, dont des réalisations sont disponibles pour chaque session, pour chaque enfant et ce, dans chaque groupe linguistique. Il est à noter que le contexte segmental de la voyelle n'a pas été pris en considération dans les analyses. L'indice sera appliqué dans un espace tridimensionnel déterminé par les fréquences des 3 premiers formants vocaliques.

\section{Résultats}

L'évolution des valeurs de PHI pour tous les participants au cours des quatre sessions est représentée sur la Figure 3. Les participants sont classés par âge chronologique initial croissant au sein de trois groupes linguistiques entourés de cadres de couleur différentes (les français-italien en bleu, les français-arabe en vert et les français-mandarin en orange). La tendance générale semble montrer que les valeurs de PHI augmentent avec l'âge pour les trois groupes linguistiques. En effet, les valeurs de PHI les plus basses sont globalement observées chez les enfants initialement âgés de moins de 26 mois - tels que la S1 des participants français-italien B09, B18 et B02 et du participant français-arabe B14 - et les plus hautes valeurs de PHI sont principalement observées chez les enfants âgés de plus de 36 mois tels que les participants français-italien B03 et B17.

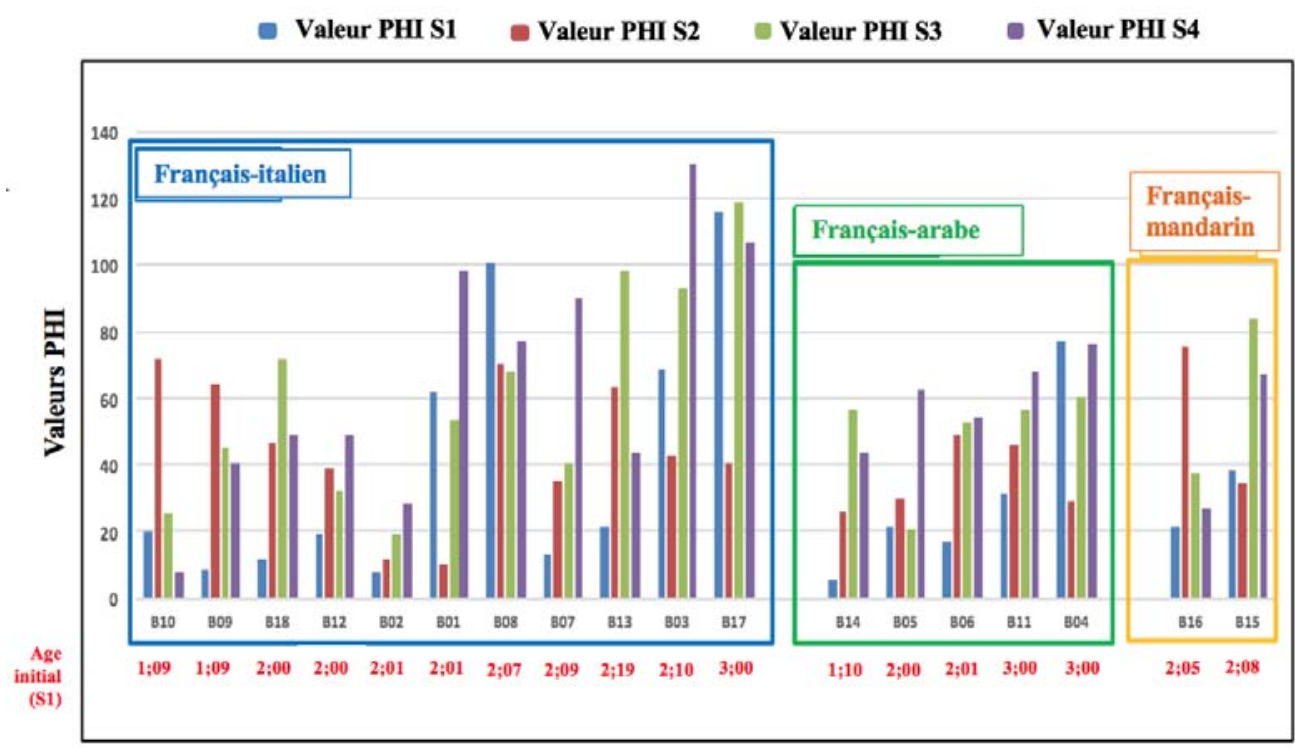

Fig. 3. Valeurs d'indice PHI pour les quatre sessions de chaque participant des trois groupes linguistiques avec les participants classés par âge chronologique initial croissant.

Toutefois, les valeurs de PHI les plus hautes ne correspondent pas forcément aux S3 et S4 des participants, puisque certains enfants présentent déjà des valeurs de $\mathrm{PHI}$ hautes lors de la S1 et/ou de la S2 (tels que B04, B08, B10, B16 et B17). Leurs valeurs de PHI redescendent ensuite durant la session suivante pour éventuellement ensuite à nouveau augmenter. Ceci suggère que le développement de l'organisation du système vocalique ne suit pas un chemin linéaire. De manière plus détaillée, la progression des valeurs de PHI à l'intérieur de chaque sujet indique une certaine variabilité pour la plupart des participants. De fait, les valeurs de PHI ont tendance à fluctuer d'une session à l'autre, particulièrement chez les plus jeunes participants (tels que B05, B10, B09, B12, B14 et B18), ce qui pourrait indiquer que l'organisation de leur système n'a pas encore atteint un état stable. Des valeurs de PHI oscillantes peuvent aussi être observées chez des enfants plus âgés (tels que B08, B03, B17 ou B04) ; toutefois, ceux-ci présentent des valeurs plus hautes et davantage de 
stabilité entre les différentes sessions. Si l'on considère les trois groupes linguistiques séparément, les valeurs de PHI les plus hautes se retrouvent chez les bilingues françaisitalien, ce qui indique qu'ils séparent mieux les catégories vocaliques $(/ \mathrm{a}, \mathrm{i}, \mathrm{u} / \mathrm{)}$ et/ou produisent les voyelles avec moins de variabilité. La Figure 4 présente une vue d'ensemble des productions des voyelles, de la session 1 à la session 4, dans les trois groupes linguistiques, tous participants confondus.
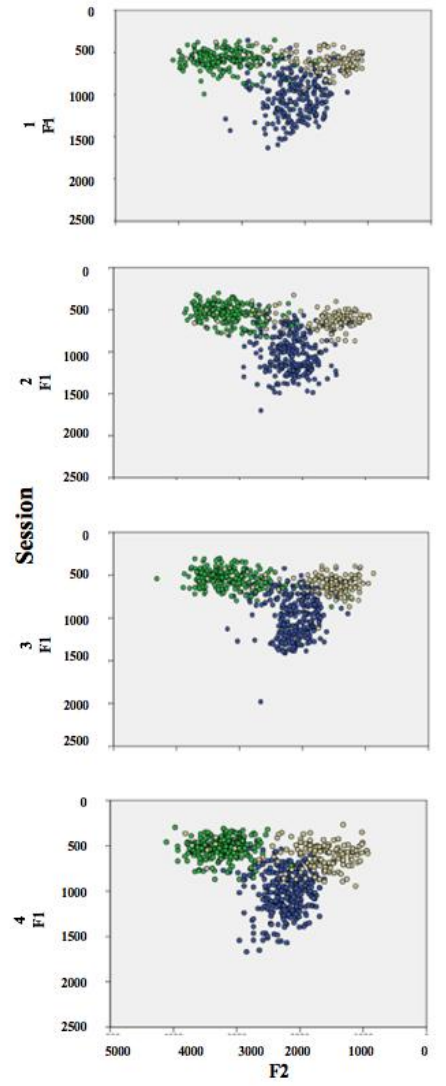

Français-italien
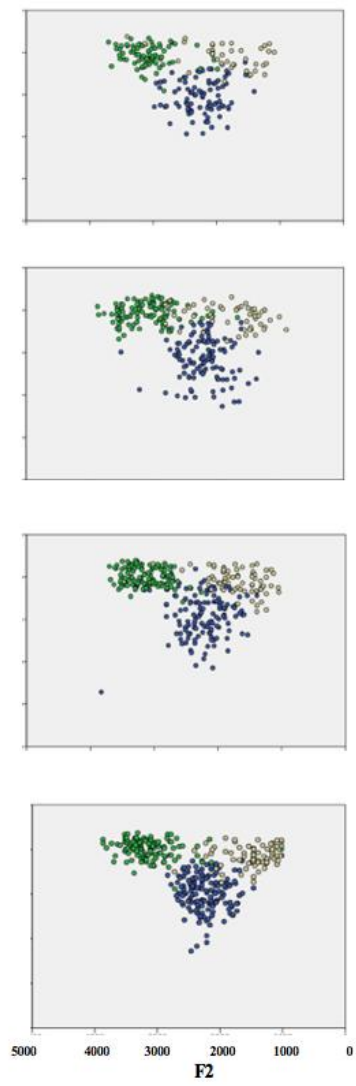

Français-arabe
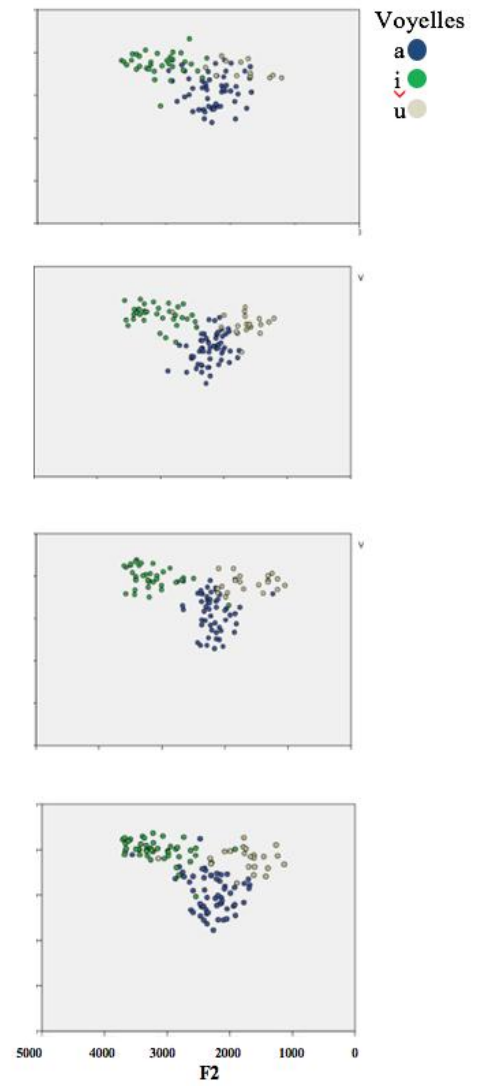

Français-mandarin

Fig. 4. Graphiques de dispersion des productions des voyelles /a/, /i/ et /u/ pour les quatre sessions de chaque groupe linguistique.

La comparaison des graphiques de dispersion des trois groupes indique une augmentation du degré d'organisation du système vocalique de la S1 à la S4 pour les trois groupes : les productions vocaliques deviennent graduellement moins dispersées et les catégories vocaliques de plus en plus distinctes les unes des autres. Les bilingues françaisitalien montrent un développement plus linéaire et présentent déjà une certaine organisation à la S1. Les bilingues français-arabe ont des performances intermédiaires à celles des deux autres groupes et présentent une régression à la $\mathrm{S} 2$ lors de laquelle les productions de voyelles deviennent plus dispersées et moins regroupées au sein des catégories. Leurs productions semblent davantage s'organiser de la S3 à la S4. De manière contrastée, les productions de voyelles des bilingues français-mandarin sont très dispersées dans un espace F1-F2 plus restreint. Ils atteignent progressivement une plus grande organisation du système de la $\mathrm{S} 1$ à la $\mathrm{S} 3$ mais on observe une régression à la $\mathrm{S} 4$, durant laquelle les 
productions redeviennent plus dispersées.

L'observation de ces graphiques de dispersion suggère que le système vocalique est plus rapidement et stablement organisé chez les participants français-italien que chez les bilingues français-arabe et français-mandarin. Etant donné que les valeurs de PHI et les graphes de dispersion suggèrent des patrons développementaux différents dans les trois groupes linguistiques, nous avons quantifié la force de la liaison entre les valeurs de PHI et l'âge chronologique pour chaque groupe. Le Tableau 2 récapitule les résultats numériques et la Figure 5 présente les graphes bivariés correspondants (étiquetées avec le numéro de la session). Ceux-ci montrent, au premier coup d'œil, un trend montant, confirmé par les valeurs de coefficient de corrélation toutes positives entre PHI et l'âge chronologique (non significative pour le groupe français-mandarin, dont l'effectif est spécialement faible).

Tableau 2. Coefficients de corrélation $\mathrm{r}_{\mathrm{BP}}$ entre les valeurs de PHI et d'âge chronologique pour chaque groupe linguistique.

\begin{tabular}{|c|c|c|c|}
\hline $\begin{array}{c}\text { Corrélation } \\
\text { PHI et } \\
\text { âge }\end{array}$ & Français-italien & Français-arabe & Français-mandarin \\
\cline { 2 - 4 } & $\mathrm{r}=0.599$ & $\mathrm{r}=0.739$ & $\mathrm{r}=0.433$ \\
chronologique & $\mathrm{p}<.001$ & $\mathrm{p}<.001$ & $\mathrm{p}=0.283$ \\
\hline
\end{tabular}
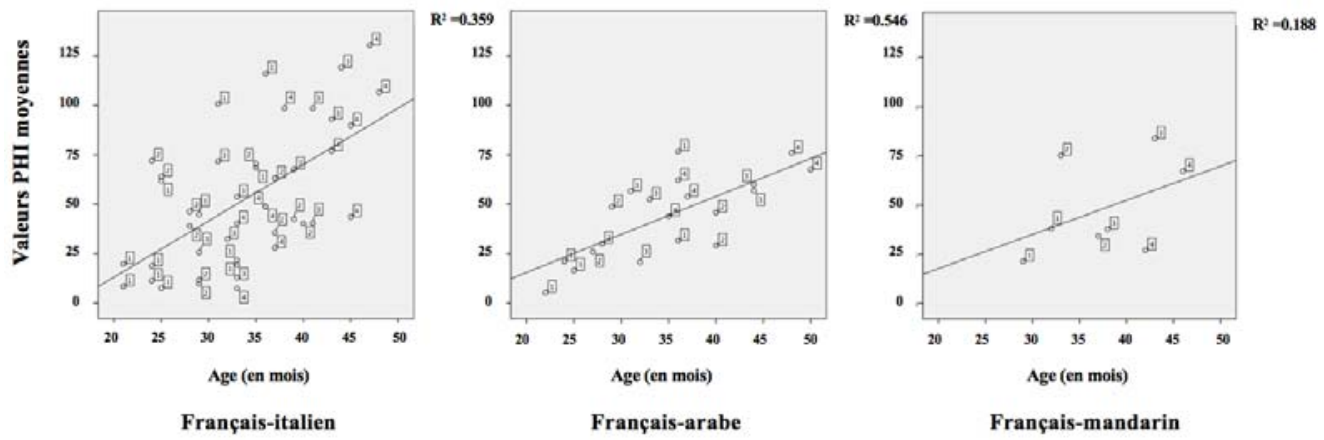

Fig. 5 Graphes de dispersion des valeurs de PHI en fonction de l'âge chronologique (en mois) pour chaque groupe linguistique.

Afin d'examiner si les trois groupes diffèrent ou se ressemblent au niveau de l'évolution de la variabilité vocalique inter- et intra-catégorie, nous avons également évalué la corrélation entre les valeurs de $\mathrm{PHI}$ et les valeurs de $\mathrm{CM}_{\text {inter }}$ et $\mathrm{CM}_{\text {intra }}$ pour chaque groupe. Les résultats sont présentés dans le Tableau 3.

Tableau 3. Coefficients de corrélation $\mathrm{r}_{\mathrm{BP}}$ entre les valeurs de PHI, $\mathrm{CM}_{\text {inter }}$ et $\mathrm{CM}_{\text {intra }}$ pour chaque groupe linguistique.

\begin{tabular}{|l|c|c|}
\hline & Corrélation PHI et $\mathrm{CM}_{\text {inter }}$ & Corrélation PHI et $\mathrm{CM}_{\text {intra }}$ \\
\hline Français-italien & $\mathrm{r}=0.820-\mathrm{p}<.001$ & $\mathrm{r}=-0.605-\mathrm{p}<.001$ \\
\hline Français-arabe & $\mathrm{r}=0.919-\mathrm{p}<.001$ & $\mathrm{r}=-0.491-\mathrm{p}=.028$ \\
\hline Français-mandarin & $\mathrm{r}=0.793-\mathrm{p}=.019$ & $\mathrm{r}=-0.516-\mathrm{p}=.191$ \\
\hline
\end{tabular}

De manière générale, on observe des corrélations positives entre $\mathrm{PHI}$ et $\mathrm{CM}_{\text {inter }}$, ce qui 
corrobore l'idée que l'éloignement des clusters vocaliques les uns des autres est un facteur de l'organisation du système, mais des corrélations négatives entre PHI et $\mathrm{CM}_{\text {intra, }}$, confirmant l'idée que la diminution de la variabilité intra-cluster est également facteur d'organisation. Toutefois, la corrélation entre les valeurs de PHI et de $\mathrm{CM}_{\text {intra }}$ est plus faible chez les bilingues français-arabe que chez les français-italien et est non significative pour les bilingues français-mandarin. La Figure 6 permet de visualiser l'évolution des valeurs de $\mathrm{CM}_{\text {inter, }}, \mathrm{CM}_{\text {intra }}$ et PHI au cours des quatre sessions dans les trois groupes linguistiques.
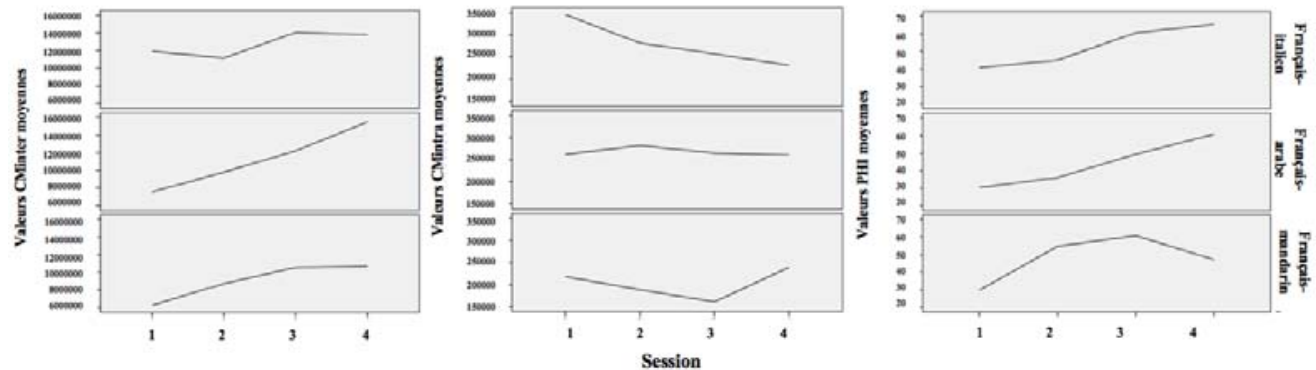

Fig. 6. Évolution des valeurs de $\mathrm{CM}_{\text {inter }}, \mathrm{CM}_{\text {intra }}$ et $\mathrm{PHI}$ au cours des quatre sessions pour chaque groupe linguistique.

Les graphiques ci-dessus montrent que les deux carrés moyens $\left(\mathrm{CM}_{\text {inter }}\right.$ et $\left.\mathrm{CM}_{\text {intra }}\right)$ évoluent différemment dans les trois groupes linguistiques. Premièrement, les bilingues françaisitalien ont initialement des valeurs de $\mathrm{CM}_{\text {inter }}$ supérieures à celles des deux autres groupes et ce, jusqu'à la S3. Ceci suggère des catégories vocaliques initialement plus distinctes les unes des autres, en lien avec les graphes de dispersion de la Figure 4. En revanche, leurs valeurs de $\mathrm{CM}_{\text {intra }}$ sont initialement plus hautes que pour les deux autres groupes et diminuent progressivement jusqu'à la $\mathrm{S} 4$, ce qui indique que leurs productions sont initialement caractérisées par une plus grande variabilité intra-catégorie. L'évolution des deux carrés moyens fait que l'on observe globalement les valeurs de PHI les plus élevées pour ce groupe. Initialement plus basses que celles des bilingues français-italien, les valeurs de $\mathrm{CM}_{\text {intra }}$ des bilingues français-arabe n'évoluent pratiquement pas de la $\mathrm{S} 1$ à la $\mathrm{S} 4$, ce qui indique que, pour ce groupe, la variabilité intra-catégorie demeure constante au cours des différentes sessions. Toutefois, l'évolution plus marquée des valeurs de $\mathrm{CM}_{\text {inter }}$ fait que les valeurs de PHI augmentent de manière linéaire entre la S2 et la S4. Enfin, on observe globalement des valeurs de $\mathrm{CM}_{\text {inter }}$ et $\mathrm{CM}_{\text {intra }}$ plus basses chez les bilingues françaismandarin, indiquant à la fois une plus grande variabilité inter-catégorie et une plus faible variabilité intra-catégorie. Ceci concorde avec les graphiques de dispersion (Fig. 4) sur lesquels on pouvait observer des productions vocaliques davantage dispersées dans une zone F1-F2 plus restreinte. Ensuite, leurs valeurs de $\mathrm{CM}_{\text {inter }}$ et $\mathrm{CM}_{\text {intra }}$ augmentent et diminuent respectivement, résultant en des valeurs de PHI croissantes. Toutefois, les valeurs de $\mathrm{CM}_{\text {intra }}$ augmentent à la $\mathrm{S} 4$, indiquant une plus grande variabilité intra-catégorie, ce qui est reflété par des valeurs de PHI descendantes. Cette régression finale pouvait également être observée dans les graphiques de dispersion (Fig. 4).

Enfin, nous avons également examiné s'il existe un lien entre les valeurs de PHI et la dominance linguistique. Aucune corrélation significative n'a été identifiée. Nous avons également testé l'hypothèse d'un lien entre les valeurs de PHI et les deux scores de vocabulaire (en français et global pour les deux langues combinées). Le Tableau 4 présente les résultats pour chaque groupe linguistique séparément. On y observe que les corrélations sont significatives pour les groupes français-italien et français-arabe, mais pas pour le groupe français-mandarin. 
Tableau 4. Coefficients de corrélation $\mathrm{r}_{\mathrm{BP}}$ entre les valeurs de PHI et les scores de vocabulaire (français/global) pour chaque groupe linguistique.

\begin{tabular}{|l|c|c|c|}
\hline & Français-italien & Français-arabe & Français-mandarin \\
\hline Corrélation PHI et score de & $\mathrm{r}=0.323$ & $\mathrm{r}=0.498$ & $\mathrm{r}=0.310$ \\
vocabulaire en français & $\mathrm{p}=0.032$ & $\mathrm{p}=0.025$ & $\mathrm{p}=0.454$ \\
\hline Corrélation PHI et score de & $\mathrm{r}=0.351$ & $\mathrm{r}=0.537$ & $\mathrm{r}=0.119$ \\
vocabulaire global & $\mathrm{p}=0.019$ & $\mathrm{p}=0.015$ & $\mathrm{p}=0.779$ \\
\hline
\end{tabular}

\section{Discussion}

Les résultats des analyses ont montré que, globalement, les valeurs de PHI augmentent avec l'âge chronologique pour l'ensemble des participants, les valeurs les plus basses étant observées chez les enfants les plus jeunes et les valeurs les plus hautes chez les enfants les plus âgés. En d'autres termes, malgré la probable réduction anatomiquement induite du champ de production acoustique, le système vocalique devient de plus en plus organisé au fur et à mesure que les enfants deviennent plus âgés. On obtient donc ici une confirmation numérisée et objectivement fondée de l'idée générale que la production vocalique s'améliore avec l'âge chronologique. Par ailleurs, ces résultats indiquent que les voyelles sont toujours en cours d'acquisition durant la tranche d'âge investiguée dans cette étude, c'est-à-dire entre 21 et 50 mois. Ceci est en accord avec les résultats de l'étude menée par Grandon (2016) mentionnée dans l'introduction, d'après lesquels les différentes catégories vocaliques seraient en voie de stabilisation entre 5 et 11 ans. Des sessions supplémentaires couvrant la tranche d'âge entre 4 et 5 ans seraient toutefois nécessaires afin de pouvoir vérifier cela, étant donné le manque de données disponibles dans la littérature.

En outre, le développement de l'organisation du système vocalique semble être non linéaire, étant donné que les valeurs de PHI fluctuent d'une session à l'autre chez la majorité des participants et ce, particulièrement chez les plus jeunes enfants. Ceci n'est pas étonnant vu la difficulté, pour l'enfant, de réaliser dynamiquement l'adaptation du contrôle de ses productions alors même que son appareil articulatoire est en incessant changement (changement dont rien ne permet de supposer le caractère linéaire). Une certaine fluctuation est également observée chez les enfants plus âgés ; toutefois, ces derniers présentent des valeurs de PHI plus hautes témoignant d'un degré d'organisation supérieur. Ces résultats suggèrent donc une grande variabilité individuelle dans le développement de l'organisation du système vocalique, comme précédemment observé dans d'autres travaux portant sur l'acquisition bilingue (Konhert, 2010 ; Kehoe \& Havy, 2019).

Les valeurs de PHI les plus hautes ont été observées chez les bilingues français-italien indiquant une meilleure distinction des catégories vocaliques /a, i, u/ et/ou des productions moins variables au sein des différentes catégories vocaliques. Ceci est confirmé par les graphes de dispersion des productions des voyelles des trois groupes linguistiques (tous participants confondus) au cours des quatre sessions qui montrent que le système vocalique du français des bilingues français-italien est initialement mieux organisé et présente un développement plus continu que celui des deux autres groupes. La plus grande similarité entre les deux langues (sur de nombreux plans: phonologique, typologique, lexical, syntaxique, etc.) pourrait être de nature facilitatrice. De manière contrastée, les bilingues français-mandarin présentent le système vocalique du français le moins bien organisé. On 
observe également dans ce groupe une régression finale à la S4, alors que les bilingues français-arabe progressent globalement le plus de la S1 à la S4. En cohérence avec ces résultats, les tests de corrélation entre les valeurs de PHI et d'âge chronologique pour chaque groupe ont montré une relation plus forte entre les deux variables pour le groupe français-arabe et une relation non significative pour les français-mandarin.

Différentes trajectoires développementales ont été observées dans les trois groupes. Premièrement, les valeurs de $\mathrm{CM}_{\text {inter }}$ et de $\mathrm{CM}_{\text {intra }}$ plus hautes observées chez les bilingues français-italien indiquent qu'ils distinguent en effet initialement mieux les catégories vocaliques tout en présentant une plus grande variabilité intra-catégorie. Les patrons développementaux observés pour le groupe français-mandarin s'expliquent plus difficilement en termes d'impact de la combinaison linguistique et sont probablement davantage attribuables à la variable sujet. Aucune corrélation n'a été décelée entre les valeurs de PHI et la dominance linguistique pour aucun des trois groupes. Il est possible que l'effet de la dominance linguistique ait été occulté par celui d'(une) autre(s) variable(s), tels que l'âge chronologique des participants. De fait, pour le groupe français-italien, les enfants présentant une dominance linguistique en français sont globalement les plus jeunes. Les tests de corrélation entre les valeurs de PHI et les scores de vocabulaire suggèrent une augmentation parallèle du degré d'organisation du système vocalique et de la taille du lexique, en cohérence avec les résultats d'autre études ayant démontré un lien entre les capacités phonologiques et lexicales chez des enfants bilingues (Scarpino, 2011 ; Kehoe \& Havy, 2019). Toutefois, la corrélation est la plus forte pour le groupe français-arabe, indiquant une plus grande relation entre l'organisation du système vocalique et le développement lexical pour ce groupe, et non significative pour les bilingues françaismandarin, probablement dû à un nombre limité de données pour ce groupe.

Globalement, on peut retenir que les résultats mis au jour indiquent une augmentation du degré d'organisation du système vocalique avec l'âge et un développement parallèle des compétences phonologique et lexicale. On observe également une évolution non linéaire ainsi qu'une grande variabilité individuelle. La comparaison des patrons développementaux dans la production vocalique chez des bilingues préscolaires exposés à différentes paires de langues indiquent des différences inter-linguistiques. Néanmoins, comme nous l'avons souligné d'entrée, cette contribution revêt un caractère exploratoire. Plusieurs des observations opérées sont à considérer avec prudence et toute velléité nomothétique doit être écartée. On retiendra cependant qu'une méthodologie articulée (depuis le recueil des productions jusqu'à l'analyse des systèmes) a ici été développée et testée, qu'elle s'est montrée apte à faire émerger des regards nouveaux sur l'acquisition des compétences phonologiques et phonétiques en français par des enfants bilingues, et qu'elle apparaît propre à étudier les spécificités différentielles de l'association, dans les combinaisons bilingues, du français avec d'autres langues. La recherche peut maintenant être poursuivie et élargie, notamment en ouvrant l'éventail des langues en contact avec le français, en équilibrant les effectifs des groupes, en assurant des tailles d'échantillons globalement plus importantes et en accroissant l'homogénéité des âges chronologiques dans chacune des sessions.

\section{Références bibliographiques}

Aicart-de Falco, S. \& Vion, M.. La mise en place du système phonologique du français chez desenfants entre trois et six ans : une étude de la production. Cahiers de Psychologie Cognitive Current Psychology of Cognition, Marseille : ADRSC, 1987, 7, pp.247-266. hal-00133459

Almeida, L. (2011). Acquisition de la structure syllabique en contexte de bilinguisme simultané portugais-français. Unpublished doctoral dissertation, University of Lisbon, Lisbon, Portugal. 
Almeida, L., Ferré, S., Morin, E., Prévost, P., Dos Santos, C., Tuller, L., \& Zebib, R. (2016). L'identification d'enfants bilingues avec Trouble Spécifique du Langage en France. In SHS Web of Conferences (Vol. 27, p. 10005). EDP Sciences.

Bigi, B. (2015). SPPAS-multi-lingual approaches to the automatic annotation of speech. The Phonetician-International Society of Phonetic Sciences, (111-112), 54-69.

Blecua-Falgueras, B., Poch-Olive, D. \& Harmegnies, B. (1993). Variaciones en la organización de la vocales del español y del catalán en function del estilo de habla, in Proceedings of the International Conference of Applied Linguistics, Granada, 1993, 97-107.

Boë, L., Ménard, L., Serkhane, J., Birkholz, P., Kröger, B., Badin, P .. \& Kielwasser, N. (2008). La croissance de l'instrument vocal : contrôle, modélisation, potentialités acoustiques et conséquences perceptives. Revue française de linguistique appliquée, vol. xiii(2), 59-80.

Boersma, P. \& Weenink, D. (2015). Praat, vers. 5.4. 01. Online: http://www.fon.hum.uva.nl/praat.

Brodeur, M. B., Dionne-Dostie, E., Montreuil, T., \& Lepage, M. (2010). The Bank of Standardized Stimuli (BOSS), a new set of 480 normative photos of objects to be used as visual stimuli in cognitive research. PloS one, 5(5), e10773.

Brulard, I. \& Carr, P. (2003). French-English bilingual acquisition of phonology: One production system or two? International Journal of Bilingualism, 7(2), 177-202.

Caselli, M. C. \& Casadio, P. (1995). Il primo vocabolario del bambino: guida all'uso del questionario MacArthur per la valutazione della comunicazione e del linguaggio nei primi anni di vita (Vol. 5). FrancoAngeli.

Chalard, M., Bonin, P., Méot, A., Boyer, B. \& Fayol, M. (2003). Objective age-of-acquisition (AoA) norms for a set of 230 object names in French - relationships with psycholinguistic variables, the English data from Morrison et al. (1997), and naming latencies. 15(2), 209-245.

Delplancq, V., Harmegnies, B. \& Poch-Olive, D. (1994). Variabilités vocaliques en portugais sous l'effet du style de parole, in Actas do IX encontro da Associação Portuguesa de Linguística, Coimbra, 1994, 165-181.

Fabiano-Smith, L., \& Barlow, J. A. (2010). Interaction in bilingual phonological acquisition: Evidence from phonetic inventories. International journal of bilingual education and bilingualism, 13(1), 81-97.

Fenson, L., Dale, P. S., Reznick, J. S., Thal, D., Bates, E., Hartung, J. P., Pethick, S. \& Reilly, J. S. (1993). MacArthur Communicative Development Inventories: User's guide and technical manual. San Diego: CA Singular Publishing Group.

Flege, J. E. (2007). Language contact in bilingualism: Phonetic system interactions. Laboratory phonology, 9, 353-382.

Grandon, B. (2016). Développement typique et atypique de la production de parole: caractéris-tiques segmentales et intelligibilité de la parole d'enfants porteurs d'un implant cochléaire et d'enfants normo-entendants de 5 à 11 ans. Unpublished doctoral dissertation, University of GrenobleAlpes, Grenoble, France.

Haidar, L. A. (2018). De la linguistique à la didactique, regards croisés en phonétique. OralitéVariabilité-Corpu. Mémoire d'Habilitation à diriger des recherches.

Harmegnies, B. \& Poch-Olive, D. (1992). A study of style-induced vowel variability: laboratory versus spontaneous speech in Spanish, Speech Communication, 11, 429-437.

Harmegnies, B., Poch-Olive, D. (1994). Formants frequencies variability in French vowels under the effect of various speaking styles, Revue de physique, C5, 509-512.

Huet, K. \& Harmegnies, B. (2000). Contribution à la quantification du degré d'organisation des systèmes vocaliques. In Actes de la XXIIè Journée d'Étude sur la Parole, 225-228.

Kail M. L'acquisition du langage repensée : les recherches inter-langues (II). In: L'année psychologique. 1983 vol. 83, n $^{\circ} 2$. pp.561-596. 
Kehoe, M. \& Havy, M. (2019). Bilingual phonological acquisition: the influence of language-internal, language-external, and lexical factors. Journal of Child Language, 46(2), 292-333.

Kern, S., \& Gayraud, F. (2010). Inventaire Français du Développement Communicatif: 8/30 mois. Les Editions de la Cigale.

Kohnert, K. (2010). Bilingual children with primary language impairment: Issues, evidence and implications for clinical actions. Journal of communication disorders, 43(6), 456-473.

MacLeod, A. A., Sutton, A., Trudeau, N., \& Thordardottir, E. (2011). The acquisition of consonants in Québécois French: A cross-sectional study of pre-school aged children. International Journal of Speech-Language Pathology, 13(2), 93-109.

Moreno-Martínez, F. J., \& Montoro, P. R. (2012). An ecological alternative to Snodgrass \& Vanderwart: 360 high quality colour images with norms for seven psycholinguistic variables. PloS one, 7(5), e37527.

Maillart, C. (2006). Le bilan articulatoire et phonologique. L'évaluation du langage et de la voix., 2651.

Paradis, J., Emmerzael, K., and Sorenson Duncan, T. (2010) Assessment of English Language Learners: Using Parent Report on First Language Development. Journal of Communication Disorders 43, 474-497.

Paradis, J. (2001). Do bilingual two-year-olds have separate phonological systems?. International Journal of Bilingualism, 5(1), 19-38.

Paradis, J. (2011) Individual Differences in Child English Second Language Acquisition: Comparing Child-Internal and Child-External Factors. Linguistic Approaches to Bilingualism, 1(3), 213-237.

Paradis, J., \& Genesee, F. (1996). Syntactic acquisition in bilingual children: Autonomous or interdependent? Studies in Second Language Acquisition, 18, 1-25

Poch, D. \& Harmegnies, B., "Variations structurelles des systèmes vocaliques en français et espagnol sous l'effet du style de parole", Journal de Physique, 1992, IV- $\underline{\text { C1, 283-286 }}$

Rvachew, S., Alhaidary, A., Mattock, K., \& Polka, L. (2008). Emergence of the corner vowels in the babble produced by infants exposed to Canadian English or Canadian French. Journal of Phonetics, 36(4), 564-577.

Rvachew, S., Mattock, K., Polka, L., \& Ménard, L. (2006). Developmental and cross-linguistic variation in the infant vowel space: The case of Canadian English and Canadian French. The Journal of the Acoustical Society of America, 120(4), 2250-2259.

Scarpino, S. E. (2011). The effects of language environment and oral language ability on phonological production proficiency in bilingual Spanish-English speaking children.Unpublished doctoral dissertation, Pensylavnia State University.

Tardif, T., Fletcher, P., Zhang, Z. X. \& Liang, W. L. (2008). Chinese communicative development inventories: User's guide and manual. Beijing: Peking University Medical Press.

Tuller, L., Messarra, C., Prévost, P., and Zebib, R. (2011) Questionnaire pour parents d'enfants bilingues, French version of the PaBiQ (COST Action IS0804, 2011). Ms., Université François Rabelais, Tours.

\section{Notes}

${ }^{1}$ Cette quantité minimum de temps d'exposition a été choisie sur base de l'Alberta Language Environment Questionnaire - ALEQ (Paradis, 2011).

${ }^{2}$ Les images proviennent principalement des bases de données de Moreno-Martinez et Montoro (2012) et Brodeur et al. (2010).

${ }^{3}$ Les répétitions ont été incluses dans les analyses afin de maximiser la quantité de données à analyser par enfant. 


\section{Annexes}

Tableau 5. Items classés par ordre de presentation (avec chacune des 8 séries dans la couleur spécifique correspondante) avec leur valeur d'indice de complexité globale.

\begin{tabular}{|c|c|c|}
\hline Ordre de présentation & Items & Indice de complexité \\
\hline Items d'entrainement & Lit-bébé - bateau & \\
\hline 1 & Coucou & 0,00 \\
\hline 2 & Langue & 0,06 \\
\hline 3 & Cheveux & 0,28 \\
\hline 4 & Nombril & 0,33 \\
\hline 5 & Pyjama & 0,44 \\
\hline 6 & Echarpe & 0,61 \\
\hline 1 & Pomme & 0,00 \\
\hline 2 & Robe & 0,00 \\
\hline 3 & Glace & 0,11 \\
\hline 4 & Souris & 0,17 \\
\hline 5 & Livre & 0,22 \\
\hline 6 & Yaourt & 0,39 \\
\hline 1 & Fleur & 0,11 \\
\hline 2 & Cadeau & 0,17 \\
\hline 3 & Porte & 0,22 \\
\hline 4 & Tortue & 0,28 \\
\hline 5 & Poisson & 0,33 \\
\hline 6 & Etoile & 0,39 \\
\hline 1 & Oiseau & 0,28 \\
\hline 2 & Chaussure & 0,28 \\
\hline 3 & Chaise & 0,33 \\
\hline 4 & Crayon & 0,33 \\
\hline 5 & Pantalon & 0,44 \\
\hline 6 & Eléphant & 0,50 \\
\hline 1 & Chien & 0,28 \\
\hline 2 & Cuillère & 0,28 \\
\hline 3 & Girafe & 0,28 \\
\hline 4 & Téléphone & 0,33 \\
\hline 5 & Parapluie & 0,56 \\
\hline 6 & Escalier & 0,67 \\
\hline 1 & Feuille & 0,00 \\
\hline 2 & Doigt & 0,11 \\
\hline 3 & Banane & 0,17 \\
\hline 4 & Panier & 0,28 \\
\hline 5 & Grenouille & 0,28 \\
\hline 6 & Arbre & 0,44 \\
\hline 1 & Train & 0,17 \\
\hline 2 & Vache & 0,22 \\
\hline 3 & Carotte & 0,28 \\
\hline 4 & Zèbre & 0,33 \\
\hline 5 & Cloche & 0,50 \\
\hline 6 & Champignon & 0,56 \\
\hline 1 & Peigne & 0,00 \\
\hline 2 & Bras & 0,11 \\
\hline 3 & Parc & 0,22 \\
\hline 4 & Fourmi & 0,28 \\
\hline 5 & Pingouin & 0,39 \\
\hline 6 & Fromage & 0,50 \\
\hline
\end{tabular}

